Molecules 2003, 8, 642-648

molecules

ISSN 1420-3049

http://www.mdpi.org

\title{
Microwave-Assisted Synthesis of Some 3,5-Arylated 2-Pyrazolines
}

\section{Davood Azarifar* and Hassan Ghasemnejad}

Department of Chemistry, Faculty of Science, Bu Ali- Sina University, Zip Code 65174, Hamadan, Iran.

*Author to whom correspondence should be addressed; e-mail azarifar@basu.ac.ir

Received: 21 February 2003; in revised form: 28 July 2003 / Accepted: 30 July 2003/ Published: 31 July 2003

\begin{abstract}
Condensation of 2-acetylnaphthalene with benzaldehydes under microwave irradiation affords chalcones which undergo facile and clean cyclizations with hydrazines $\mathrm{RNHNH}_{2}(\mathrm{R}=\mathrm{H}, \mathrm{Ph}, \mathrm{Ac})$ to afford 3,5-arylated 2-pyrazolines in quantitative yields, also under microwave irradiation and in the presence of dry $\mathrm{AcOH}$ as cyclizing agent. The results obtained indicate that, unlike classical heating, microwave irradiation results in higher yields, shorter reaction times (2-12 min.) and cleaner reactions.
\end{abstract}

Keywords: Microwave irradiation; chalcones; 3,5-arylated-2-pyrazolines; pyrazolines; heterocyclic synthesis.

\section{Introduction}

Variously substituted pyrazolines and their derivatives are important biological agents and a significant amount of research activity has been directed towards this class. In particular, they are used as antitumor [1], antibacterial, antifungal, antiviral, antiparasitic, anti-tubercular and insecticidal agents [2-10]. Some of these compounds have also anti-inflammatory, anti-diabetic, anaesthetic and analgesic properties [11-14]. Moreover, pyrazolines have played a crucial part in the development of theory in heterocyclic chemistry and also used extensively as useful synthons in organic synthesis [15-19]. A classical synthesis of these compounds involves the base-catalyzed aldol condensation reaction of 
aromatic ketones and aldehydes to give $\alpha, \beta$-unsaturated ketones (chalcones), which undergo a subsequent cyclization reaction with hydrazines affording 2-pyrazolines [11, 20-22]. In this method, hydrazones are formed as intermediates, which can be subsequently cyclized to 2-pyrazolines in the presence of a suitable cyclizing reagent like acetic acid [23,24].

In recent years, a significant portion of research in heterocyclic chemistry has been devoted to 2-pyrazolines containing different aryl groups as substituents, as evident from the literature [25-33]. We have recently reported on the synthesis of some newly 3,5-naphthylated 2-pyrazolines which exhibit efficient antimicrobial activity against a variety of test organisms [8].

\section{Results and Discussion}

As a result of our studies related to the development of synthetic protocols using microwave irradiation, we now report a novel and easy access to 3,5-arylated 2-pyrazolines using a one-pot procedure and demonstrate its superiority over our previously reported classical heating method [8]. We report in this paper some aldol condensation reactions between 2-acetylnaphthalene (1) and benzaldehydes 2a-e in the presence of $\mathrm{KOC}_{2} \mathrm{H}_{5} / \mathrm{C}_{2} \mathrm{H}_{5} \mathrm{OH}$ to give intermediate chalcones 3a-e which undergo a rapid cyclization with hydrazines $\mathbf{4 f}-\mathbf{h}$ under microwave irradiation at $80{ }^{\circ} \mathrm{C}$ to yield 2-pyrazolines 5af-eh quantitatively in 2-12 minutes (Scheme 1). The heterocyclic products were characterized on the basis of their IR, ${ }^{1} \mathrm{H}-\mathrm{NMR},{ }^{13} \mathrm{C}-\mathrm{NMR}$, MS spectral and elemental analysis (Table 2).

\section{Scheme 1}
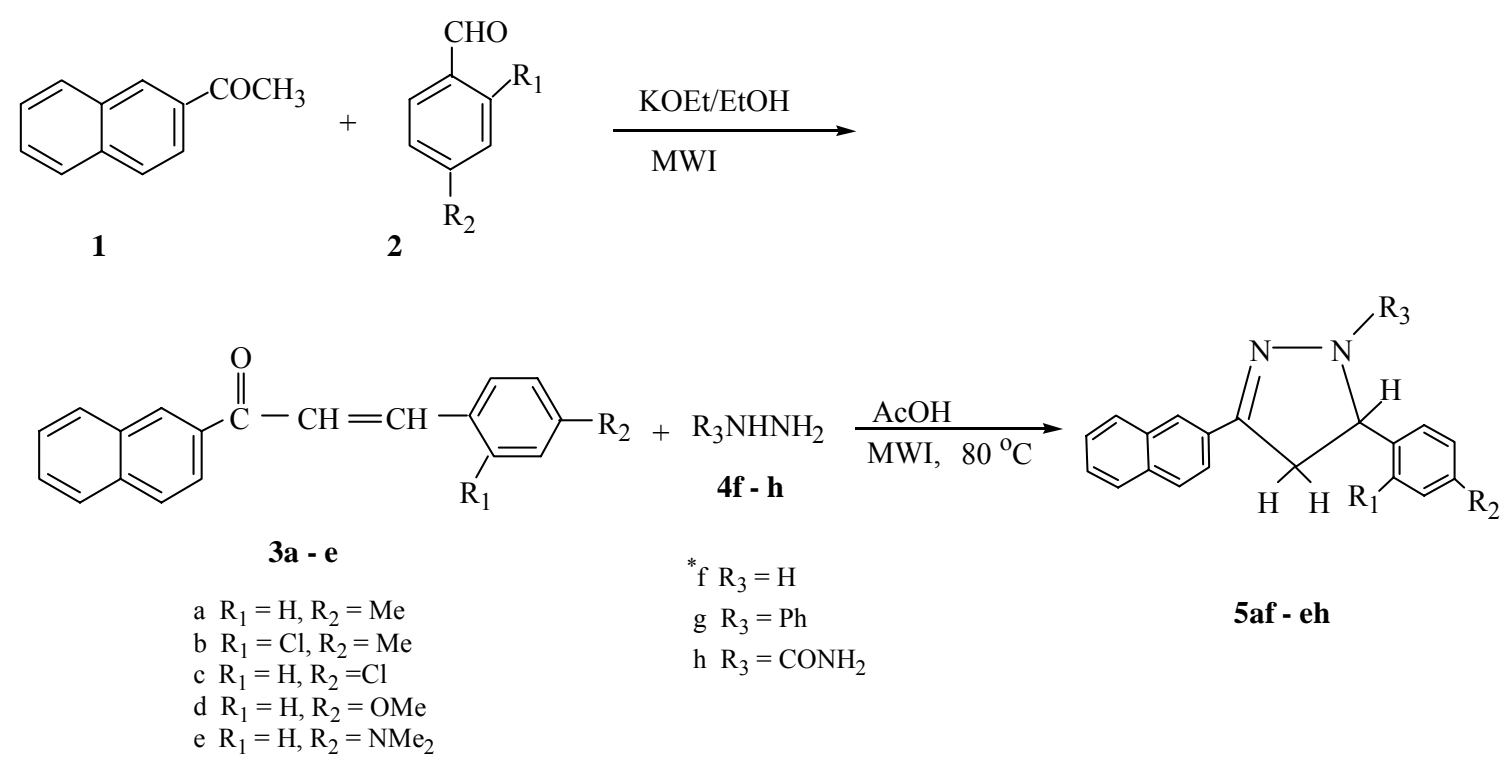

\footnotetext{
* Acetylation, in situ, occurs in the presence of $\mathrm{AcOH}$ to convert the initially formed 2-pyrazolines $\left(\mathrm{R}_{3}=\mathrm{H}\right)$ mainly into their acetylated derivatives $\left(\mathrm{R}_{3}=\mathrm{COCH}_{3}\right)$.
} 


\section{Conclusions}

In summary, this work demonstrates a rapid, efficient and environmentally friendly method of synthesis of 3,5-arylated 2-pyrazolines under microwave heating, and the results obtained confirm the superiority of the microwave irradiation method over the classical heating one.

\section{Acknowledgements}

The authors wish to acknowledge the financial support of the Bu-Ali Sina University, Chemistry Department, to carry out this research.

\section{Experimental}

\section{General}

All melting points were determined on a Büchi 530 melting point apparatus, and are uncorrected. The ${ }^{1} \mathrm{H}-\mathrm{NMR}$ spectra were recorded for deuteriochloroform solutions using tetramethylsilane as the internal standard on Jeol FX (at $90 \mathrm{MHz}$ ) and Brüker AM (at $200 \mathrm{MHz}$ ) spectrometers at ambient temperature. IR spectra were recorded on a Shimadzu IR-435U-04 instrument using potassium bromide pellets. Elemental analyses were performed at the Iran Polymer Research Center, Karaj, Iran.

General procedure for microwave-assisted preparation of 3,5-diaryl-2-pyrazoline derivatives (5af-eh).

In the first step the chalcones 3a-e are prepared by the reaction between 2-acetylnaphthalene $(1.70 \mathrm{~g}$, $10 \mathrm{mmol})$, dissolved in $1 \mathrm{M} \mathrm{KOC}_{2} \mathrm{H}_{5} / \mathrm{C}_{2} \mathrm{H}_{5} \mathrm{OH}$ solution $(10 \mathrm{~mL})$, and the corresponding benzaldehyde (10 mmol) under microwave irradiation (300 watt) for a few min. The resulting crude yellow solid is filtered, washed successively with dilute $\mathrm{HCl}$ solution and distilled water and finally recrystallized from ethanol (95\%) to give the pure chalcones 3a-e in $80-95 \%$ yield. In the second step, the hydrazine reagent $(12 \mathrm{mmol})$ is then added dropwise to a stirring solution of the chalcones 3a-e $(10 \mathrm{mmol})$ in glacial $\mathrm{AcOH}(10 \mathrm{~mL})$. The mixture is subjected to microwave heating for several min. using a domestic microwave oven (300 watt) to afford 2-pyrazolines, which were recrystallized from ethanol (95\%) to give pure compounds 5af-eh with a 82-99\% yield. Analytical data for the prepared compounds is given in Table 1. Spectroscopic data is summarized in Table 2. 
Table 1. Microwave synthesis of 3,5-arylated 2-pyrazolines (5af-eh) $($ power $=300 \mathrm{~W})$

\begin{tabular}{|c|c|c|c|c|c|c|c|}
\hline \multirow[t]{2}{*}{ Compound } & \multirow{2}{*}{$\begin{array}{c}\text { Molecular } \\
\text { Formula }\end{array}$} & \multirow{2}{*}{$\begin{array}{c}\text { Irradiation } \\
\text { Time } \\
(\text { min) }\end{array}$} & \multirow{2}{*}{$\begin{array}{l}\text { Yield }^{\mathrm{a}} \\
(\%)\end{array}$} & \multirow{2}{*}{$\begin{array}{l}\text { M.P. } \\
\left({ }^{\circ} \mathrm{C}\right)\end{array}$} & \multicolumn{3}{|c|}{$\begin{array}{c}\text { Elemental Analysis } \\
\text { Calcd (found) }\end{array}$} \\
\hline & & & & & C & $\mathbf{H}$ & $\mathbf{N}$ \\
\hline 5 af & $\mathrm{C}_{22} \mathrm{H}_{20} \mathrm{ON}_{2}$ & 7 & 85 & $\begin{array}{c}175- \\
176\end{array}$ & $\begin{array}{c}80.49 \\
(80.71)\end{array}$ & $\begin{array}{r}6.10 \\
(6.29)\end{array}$ & $\begin{array}{c}8.54 \\
(8.59)\end{array}$ \\
\hline $5 a g$ & $\mathrm{C}_{26} \mathrm{H}_{22} \mathrm{~N}_{2}$ & 1.4 & 98 & $\begin{array}{c}167- \\
168\end{array}$ & $\begin{array}{c}86.19 \\
(86.35)\end{array}$ & $\begin{array}{c}6.08 \\
(6.04)\end{array}$ & $\begin{array}{c}7.73 \\
(7.82)\end{array}$ \\
\hline 5 ah & $\mathrm{C}_{21} \mathrm{H}_{19} \mathrm{ON}_{3}$ & 7 & 88 & $\begin{array}{c}182- \\
183\end{array}$ & $\begin{array}{c}76.60 \\
(76.74)\end{array}$ & $\begin{array}{c}5.77 \\
(5.84)\end{array}$ & $\begin{array}{r}12.76 \\
(12.72)\end{array}$ \\
\hline $5 b f$ & $\mathrm{C}_{22} \mathrm{H}_{19} \mathrm{ON}_{2} \mathrm{Cl}$ & 2.3 & 96 & $\begin{array}{c}172- \\
173\end{array}$ & $\begin{array}{c}72.83 \\
(72.70)\end{array}$ & $\begin{array}{c}5.24 \\
(5.29)\end{array}$ & $\begin{array}{c}7.72 \\
(7.54)\end{array}$ \\
\hline $5 b g$ & $\mathrm{C}_{26} \mathrm{H}_{21} \mathrm{~N}_{2} \mathrm{Cl}$ & 1.7 & 98 & $\begin{array}{c}131- \\
132\end{array}$ & $\begin{array}{c}78.69 \\
(78.99)\end{array}$ & $\begin{array}{c}5.30 \\
(5.43)\end{array}$ & $\begin{array}{c}7.06 \\
(6.83)\end{array}$ \\
\hline $5 \mathrm{bh}$ & $\begin{array}{c}\mathrm{C}_{21} \mathrm{H}_{18} \mathrm{ON}_{3} \\
\mathrm{Cl}\end{array}$ & 6.4 & 82 & $\begin{array}{l}162- \\
164\end{array}$ & $\begin{array}{c}69.32 \\
(69.38)\end{array}$ & $\begin{array}{c}4.95 \\
(4.82)\end{array}$ & $\begin{array}{c}11.55 \\
(11.54)\end{array}$ \\
\hline $5 \mathrm{cf}$ & $\mathrm{C}_{21} \mathrm{H}_{17} \mathrm{ON}_{2} \mathrm{Cl}$ & 1.5 & 99 & $\begin{array}{c}177- \\
178\end{array}$ & $\begin{array}{c}72.31 \\
(72.34)\end{array}$ & $\begin{array}{c}4.88 \\
(4.85)\end{array}$ & $\begin{array}{c}8.03 \\
(8.16)\end{array}$ \\
\hline $5 \mathrm{cg}$ & $\mathrm{C}_{25} \mathrm{H}_{19} \mathrm{~N}_{2} \mathrm{Cl}$ & 1.4 & 98 & $\begin{array}{c}129- \\
130\end{array}$ & $\begin{array}{c}78.43 \\
(78.58)\end{array}$ & $\begin{array}{c}4.97 \\
(5.12)\end{array}$ & $\begin{array}{c}7.32 \\
(7.52)\end{array}$ \\
\hline $5 \mathrm{ch}$ & $\mathrm{C}_{20} \mathrm{H}_{16} \mathrm{ON}_{3} \mathrm{Cl}$ & 11 & 85 & $\begin{array}{l}180- \\
182\end{array}$ & $\begin{array}{c}68.67 \\
(68.87)\end{array}$ & $\begin{array}{c}4.58 \\
(4.67)\end{array}$ & $\begin{array}{c}12.02 \\
(11.88)\end{array}$ \\
\hline $5 \mathrm{df}$ & $\mathrm{C}_{22} \mathrm{H}_{20} \mathrm{O}_{2} \mathrm{~N}_{2}$ & 1.3 & 98 & $\begin{array}{l}186- \\
187\end{array}$ & $\begin{array}{c}76.74 \\
(76.93)\end{array}$ & $\begin{array}{c}5.81 \\
(5.59)\end{array}$ & $\begin{array}{c}8.14 \\
(8.54)\end{array}$ \\
\hline $5 \mathrm{dg}$ & $\mathrm{C}_{26} \mathrm{H}_{22} \mathrm{ON}_{2}$ & 1.2 & 98 & $\begin{array}{c}135- \\
136\end{array}$ & $\begin{array}{c}82.54 \\
(82.51)\end{array}$ & $\begin{array}{c}5.82 \\
(5.72)\end{array}$ & $\begin{array}{c}7.41 \\
(7.48)\end{array}$ \\
\hline $5 \mathrm{dh}$ & $\mathrm{C}_{21} \mathrm{H}_{19} \mathrm{O}_{2} \mathrm{~N}_{3}$ & 7.5 & 86 & $\begin{array}{l}173- \\
175\end{array}$ & $\begin{array}{c}73.04 \\
(73.12)\end{array}$ & $\begin{array}{c}5.51 \\
(5.46)\end{array}$ & $\begin{array}{c}12.17 \\
(12.21)\end{array}$ \\
\hline $5 \mathrm{ef}$ & $\mathrm{C}_{23} \mathrm{H}_{23} \mathrm{ON}_{3}$ & 4.5 & 85 & $\begin{array}{c}183- \\
184\end{array}$ & $\begin{array}{c}77.31 \\
(77.42)\end{array}$ & $\begin{array}{c}6.44 \\
(6.48)\end{array}$ & $\begin{array}{r}11.76 \\
(11.79)\end{array}$ \\
\hline $5 \mathrm{eg}$ & $\mathrm{C}_{27} \mathrm{H}_{25} \mathrm{~N}_{3}$ & 2.2 & 99 & $\begin{array}{c}185- \\
186\end{array}$ & $\begin{array}{c}82.86 \\
(82.83)\end{array}$ & $\begin{array}{c}6.39 \\
(6.41)\end{array}$ & $\begin{array}{c}10.74 \\
(10.78)\end{array}$ \\
\hline $5 \mathrm{eh}$ & $\mathrm{C}_{22} \mathrm{H}_{22} \mathrm{ON}_{4}$ & 6.8 & 86 & $\begin{array}{c}192- \\
193 \\
\end{array}$ & $\begin{array}{r}73.74 \\
(73.87) \\
\end{array}$ & $\begin{array}{c}6.14 \\
(6.34)\end{array}$ & $\begin{array}{r}15.64 \\
(15.28)\end{array}$ \\
\hline
\end{tabular}

${ }^{\mathrm{a}}$ Yields of isolated products, calculated on the basis of the chalcones 3a-e. 
Table 2. IR, ${ }^{1} \mathrm{H}-\mathrm{NMR}$ and MS (EI) spectral data of the 2-pyrazoline products

\begin{tabular}{|c|c|c|c|}
\hline Compound & IR $\left(\mathrm{cm}^{-1}\right)$ & ${ }^{1} \mathrm{H}-\mathrm{NMR}$ (ppm) & $\operatorname{MS}(\mathbf{m} / \mathbf{z})$ \\
\hline 5af & $\begin{array}{l}3068,2975,1660 \\
1620,1580,1484 \\
1337,1638,806\end{array}$ & $\begin{array}{l}2.38(\mathrm{~s}, 3 \mathrm{H}, \mathrm{Me}), 2.46(\mathrm{~s}, 3 \mathrm{H}, \mathrm{COMe}), 3.16(\mathrm{dd}, J= \\
\left.18.2,5.1 \mathrm{~Hz}, 1 \mathrm{H}, \mathrm{CH}_{2(\text { Pyraz) }}\right), 3.80(\mathrm{dd}, J=18.2,11.8 \\
\left.\mathrm{Hz}, 1 \mathrm{H}, \mathrm{CH}_{(\text {Pyraz }}\right), 5.91(\mathrm{dd}, J=11.8,5.1 \mathrm{~Hz}, 1 \mathrm{H}, \\
\left.\mathrm{CH}_{2 \text { (Pyraz) }}\right), 7.12(\mathrm{~s}, 4 \mathrm{H}, \mathrm{Ph}), 7.25-8.00(\mathrm{~m}, 7 \mathrm{H}, \\
\mathrm{Naph})\end{array}$ & $\begin{array}{l}57,71,77,91,127,153 \\
255,285,286,328,329\end{array}$ \\
\hline 5ag & $\begin{array}{l}3178,2938, \\
2813,1600,1500 \\
1100,870,820,750\end{array}$ & $\begin{array}{l}2.40(\mathrm{~s}, 3 \mathrm{H}, \mathrm{Me}), 3.05(\mathrm{dd}, J=16.9,8.4 \mathrm{~Hz}, 1 \mathrm{H}, \\
\left.\mathrm{CH}_{2(\text { (yraz) }}\right), 3.82\left(\mathrm{dd}, J=16.9,12.0 \mathrm{~Hz}, 1 \mathrm{H}, \mathrm{CH}_{\text {Pyraz }}\right) \\
5.29\left(\mathrm{dd}, J=12.0,8.4 \mathrm{~Hz}, 1 \mathrm{H}, \mathrm{CH}_{2(\text { (Prraz) }}\right), 6.60- \\
8.20(\mathrm{~m}, 16 \mathrm{H}, \mathrm{Ar})\end{array}$ & $\begin{array}{l}42,77,91,101,118,125, \\
127,153,167,244,271,362\end{array}$ \\
\hline 5ah & $\begin{array}{l}3500,3350,3115 \\
2985,1675,1580 \\
1500,1160,855,760\end{array}$ & $\begin{array}{l}2.23(\mathrm{~s}, 3 \mathrm{H}, \mathrm{Me}), 3.26(\mathrm{dd}, J=18.3,5.8 \mathrm{~Hz}, 1 \mathrm{H}, \\
\left.\mathrm{CH}_{2(\text { Pyraz })}\right), 3.77\left(\mathrm{dd}, J=18.3,12.4 \mathrm{~Hz}, 1 \mathrm{H}, \mathrm{CH}_{\text {Pyraz }},\right. \\
5.33\left(\mathrm{bs}, 2 \mathrm{H}, \mathrm{NH}_{2}\right), 5.50(\mathrm{dd}, J=12.4,5.8 \mathrm{~Hz}, 1 \mathrm{H}, \\
\left.\mathrm{CH}_{2 \text { (Pyraz) }}\right), 7.08(\mathrm{~s}, 4 \mathrm{H}, \mathrm{Ph}), 7.20-8.00(\mathrm{~m}, 7 \mathrm{H}, \\
\mathrm{Naph})\end{array}$ & $\begin{array}{l}42,77,91,101,127,169 \\
153,118,195,238,285 \\
313,329\end{array}$ \\
\hline 5bf & $\begin{array}{l}3065,2915,1670 \\
1590,1470,1450, \\
1330,1150,975,865 \\
745,630\end{array}$ & $\begin{array}{l}2.42(\mathrm{~s}, 3 \mathrm{H}, \mathrm{Me}), 2.52(\mathrm{~s}, 3 \mathrm{H}, \mathrm{COMe}), 3.16(\mathrm{dd}, J= \\
\left.18.0,5.2 \mathrm{~Hz}, 1 \mathrm{H}, \mathrm{CH}_{2(\text { (yraz) }}\right), 3.83(\mathrm{dd}, J=18.0,12.0 \\
\left.\mathrm{Hz}, 1 \mathrm{H}, \mathrm{CH}_{\text {Pyraz }}\right), 5.92(\mathrm{dd}, J=12.0,5.20 \mathrm{~Hz}, 1 \mathrm{H}, \\
\left.\mathrm{CH}_{2 \text { (Pyraz) }}\right), 7.10-8.10(\mathrm{~m}, 10 \mathrm{H}, \mathrm{Ar})\end{array}$ & $\begin{array}{l}57,71,77,112,127,138 \\
154,271,237,277,279 \\
294,292,305,307,308 \\
348,350\end{array}$ \\
\hline 5bg & $\begin{array}{l}3058,2883,1594 \\
1500,1460,1325 \\
1120,1050,865,745 \\
700\end{array}$ & $\begin{array}{l}2.38(\mathrm{~s}, 3 \mathrm{H}, \mathrm{Me}), 3.14(\mathrm{dd}, J=16.6,8.1 \mathrm{~Hz}, 1 \mathrm{H}, \\
\left.\mathrm{CH}_{2(\text { (yraz) }}\right), 4.11\left(\mathrm{dd}, J=16.6,11.6 \mathrm{~Hz}, 1 \mathrm{H}, \mathrm{CH}_{\text {Pyraz }}\right) \\
5.58\left(\mathrm{dd}, J=11.6,8.1 \mathrm{~Hz}, 1 \mathrm{H}, \mathrm{CH}_{2(\text { (Prraz) }}\right), 6.50- \\
8.20(\mathrm{~m}, 15 \mathrm{H}, \mathrm{Ar})\end{array}$ & $\begin{array}{l}77,91,101,113,111,127 \\
138,140,153,167,244 \\
271,382,384\end{array}$ \\
\hline 5bh & $\begin{array}{l}3450,3250,3085 \\
2965,1680,1580 \\
1478,1240,1075 \\
820,750\end{array}$ & $\begin{array}{l}2.28(\mathrm{~s}, 3 \mathrm{H}, \mathrm{Me}), 3.33(\mathrm{dd}, J=16.6,5.8 \mathrm{~Hz}, 1 \mathrm{H}, \\
\left.\mathrm{CH}_{2 \text { (Pyraz) }}\right), 3.89\left(\mathrm{dd}, J=16.6,12.2 \mathrm{~Hz}, 1 \mathrm{H}, \mathrm{CH}_{\text {Pyraz }},\right. \\
5.38\left(\mathrm{bs}, 2 \mathrm{H}, \mathrm{NH}_{2}\right), 5.60(\mathrm{dd}, J=12.2,5.8 \mathrm{~Hz}, 1 \mathrm{H}, \\
\left.\mathrm{CH}_{2 \text { (Pyraz) }}\right), 7.26(\mathrm{~s}, 3 \mathrm{H}, \mathrm{Ph}), 7.38-8.20(\mathrm{~m}, 7 \mathrm{H}, \\
\mathrm{Naph})\end{array}$ & $\begin{array}{l}42,91,115,127,153,169 \\
196,195,249,305,307 \\
349,351\end{array}$ \\
\hline $5 \mathrm{cf}$ & $\begin{array}{l}3048,2980,1665 \\
1600,1500,1475 \\
1452,1195,986,735 \\
640\end{array}$ & $\begin{array}{l}2.42(\mathrm{~s}, 3 \mathrm{H}, \mathrm{COMe}), 3.12(\mathrm{dd}, J=18.0,6.20 \mathrm{~Hz}, 1 \mathrm{H}, \\
\left.\mathrm{CH}_{2(\text { Pyraz }}\right), 3.78\left(\mathrm{dd}, J=18.0,12.6 \mathrm{~Hz}, 1 \mathrm{H}, \mathrm{CH}_{\text {Pyraz }}\right) \\
5.95\left(\mathrm{dd}, J=18.0,6.2 \mathrm{~Hz}, 1 \mathrm{H}, \mathrm{CH}_{2 \text { (Pyraz) }}\right), 7.20(\mathrm{~s}, \\
4 \mathrm{H}, \mathrm{Ph}), 7.32-8.30(\mathrm{~m}, 7 \mathrm{H}, \mathrm{Naph})\end{array}$ & $\begin{array}{l}57,77,114,127,138,153 \\
271,237,277,276,294 \\
292,306,307,308,348 \\
349,350\end{array}$ \\
\hline $5 \mathrm{cg}$ & $\begin{array}{l}3072,2918,1600 \\
1500,1360,1135 \\
825,730\end{array}$ & $\begin{array}{l}3.18\left(\mathrm{dd}, J=16.6,8.5 \mathrm{~Hz}, 1 \mathrm{H}, \mathrm{CH}_{2 \text { (Pyraz) }}\right), 3.83 \\
\left(\mathrm{dd}, J=16.6,11.4 \mathrm{~Hz}, 1 \mathrm{H}, \mathrm{CH}_{\text {Pyraz }}\right), 5.18(\mathrm{dd}, J= \\
\left.11.4,8.5 \mathrm{~Hz}, 1 \mathrm{H}, \mathrm{CH}_{2(\text { (Pyraz) }}\right), 6.60-8.20(\mathrm{~m}, 16 \mathrm{H}, \\
\text { Ar) }\end{array}$ & $\begin{array}{l}77,91,101,113,127,139 \\
140,153,168,244,271 \\
382,383,384\end{array}$ \\
\hline $5 \mathrm{ch}$ & $\begin{array}{l}3420,3300,3120 \\
2900,1675,1582 \\
1487,1500,1220 \\
1085,820,745\end{array}$ & $\begin{array}{l}3.25\left(\mathrm{dd}, J=17.4,6.4 \mathrm{~Hz}, 1 \mathrm{H}, \mathrm{CH}_{2 \text { (Pyraz) }}\right), 3.81(\mathrm{dd}, J \\
\left.=17.4,12.2 \mathrm{~Hz}, 1 \mathrm{H}, \mathrm{CH}_{\text {Pyraz }}\right), 5.35\left(\mathrm{bs}, 2 \mathrm{H}, \mathrm{NH}_{2}\right), \\
5.48\left(\mathrm{dd}, J=12.2,6.4 \mathrm{~Hz}, 1 \mathrm{H}, \mathrm{CH}_{2 \text { (Pyraz) }}\right), 7.18(\mathrm{~s}, \\
4 \mathrm{H}, \mathrm{Ph}), 7.30-8.10(\mathrm{~m}, 7 \mathrm{H}, \mathrm{Naph})\end{array}$ & $\begin{array}{l}42,77,101,115,127,153 \\
169,196,195,228,293 \\
305,307,349,351\end{array}$ \\
\hline $5 \mathrm{df}$ & $\begin{array}{l}3068,2887,2838 \\
1670,1600,1495 \\
1448,1120,1095 \\
954,740\end{array}$ & $\begin{array}{l}2.45(\mathrm{~s}, 3 \mathrm{H}, \mathrm{COMe}), 3.25(\mathrm{dd}, J=18.1,5.8 \mathrm{~Hz}, 1 \mathrm{H}, \\
\left.\mathrm{CH}_{2 \text { (Pyraz) }}\right), 3.75(\mathrm{~s}, 3 \mathrm{H}, \mathrm{OMe}), 3.82(\mathrm{dd}, J=18.1, \\
\left.12.2 \mathrm{~Hz}, 1 \mathrm{H}, \mathrm{CH}_{\mathrm{Pyraz}}\right), 5.54(\mathrm{dd}, J=12.2,5.8 \mathrm{~Hz}, 1 \mathrm{H}, \\
\left.\mathrm{CH}_{2 \text { (Pyraz) }}\right), 6.82\left(\mathrm{~d}, J=9.5 \mathrm{~Hz}, 2 \mathrm{H}, 3-\mathrm{H}_{\mathrm{Ph}}\right), 7.20(\mathrm{~d}, J \\
\left.=9.5 \mathrm{~Hz}, 2 \mathrm{H}, 2-\mathrm{H}_{\mathrm{Ph}}\right), 7.50-8.10(\mathrm{~m}, 7 \mathrm{H}, \mathrm{Naph})\end{array}$ & $\begin{array}{l}57,77,101,108,127,134 \\
153,167,274,301,329 \\
344,345\end{array}$ \\
\hline
\end{tabular}


Table 2. Cont.

\begin{tabular}{|c|c|c|c|}
\hline Compounds & IR $\left(\mathrm{cm}^{-1}\right)$ & ${ }^{1} \mathrm{H}-\mathrm{NMR}$ (ppm) & $\operatorname{MS}(\mathbf{m} / \mathbf{z})$ \\
\hline $5 d g$ & $\begin{array}{l}3150,2880,1600 \\
1495,1410,1350 \\
1240,1115,1035 \\
820,740\end{array}$ & $\begin{array}{l}3.29\left(\mathrm{dd}, J=17.8,6.0 \mathrm{~Hz}, 1 \mathrm{H}, \mathrm{CH}_{2(\text { Pyraz })}\right), 3.82(\mathrm{dd}, J \\
\left.=17.8,12.0 \mathrm{~Hz}, 1 \mathrm{H}, \mathrm{CH}_{\text {Pyraz }}\right), 3.70(\mathrm{~s}, 3 \mathrm{H}, \mathrm{Me}), 5.18 \\
\left(\mathrm{dd}, J=12.0,6.0 \mathrm{~Hz}, 1 \mathrm{H}, \mathrm{CH}_{2(\text { (Prraz })}\right), 6.60-8.20(\mathrm{~m}, \\
16 \mathrm{H}, \mathrm{Ar})\end{array}$ & $\begin{array}{l}77,107,127,134,153,154, \\
167,244,271,378\end{array}$ \\
\hline $5 \mathrm{dh}$ & $\begin{array}{l}3480,3375,3150 \\
2900,1685,1580 \\
1520,1490,1250 \\
1070,865,750\end{array}$ & $\begin{array}{l}3.27\left(\mathrm{dd}, J=18.1,5.0 \mathrm{~Hz}, 1 \mathrm{H}, \mathrm{CH}_{2(\text { Pyraz })}\right), 3.69(\mathrm{~s} \\
3 \mathrm{H}, \mathrm{Me}), 3.78\left(\mathrm{dd}, J=18.1,11.2 \mathrm{~Hz}, 1 \mathrm{H}, \mathrm{CH}_{\text {Pyraz }}\right) \\
5.40\left(\mathrm{bs}, 2 \mathrm{H}, \mathrm{NH}_{2}\right), 5.52(\mathrm{dd}, J=11.2,5.0 \mathrm{~Hz}, 1 \mathrm{H}, \\
\left.\mathrm{CH}_{2(\text { Pyraz })}\right), 6.77\left(\mathrm{~d}, J=9.8 \mathrm{~Hz}, 2 \mathrm{H}, 3-\mathrm{H}_{\mathrm{Ph}}\right), 7.15(\mathrm{~d}, \\
\left.J=9.8 \mathrm{~Hz}, 2 \mathrm{H}, 2-\mathrm{H}_{\mathrm{Ph}}\right), 7.30-8.00(\mathrm{~m}, 7 \mathrm{H}, \mathrm{Naph})\end{array}$ & $\begin{array}{l}77,91,121,134,149,153 \\
169,191,195,303,302 \\
345,346\end{array}$ \\
\hline $5 \mathrm{ef}$ & $\begin{array}{l}3087,2983,2810 \\
1657,1648,1615 \\
1595,1340,1075 \\
815\end{array}$ & $\begin{array}{l}2.43(\mathrm{~s}, 3 \mathrm{H}, \mathrm{COMe}), 2.83\left(\mathrm{~s}, 6 \mathrm{H}, \mathrm{NMe}_{2}\right), 3.12(\mathrm{dd}, J \\
\left.=15.9,7.9 \mathrm{~Hz}, 1 \mathrm{H}, \mathrm{CH}_{2(\text { Pyraz })}\right), 3.75(\mathrm{dd}, J=15.9, \\
\left.11.8 \mathrm{~Hz}, 1 \mathrm{H}, \mathrm{CH}_{\text {Pyraz }}\right), 5.85(\mathrm{dd}, J=11.8,7.9 \mathrm{~Hz}, \\
\left.1 \mathrm{H}, \mathrm{CH}_{2(\text { Pyraz }}\right), 7.35\left(\mathrm{~d}, J=9.6 \mathrm{~Hz}, 2 \mathrm{H}, 3-\mathrm{H}_{\mathrm{Ph}}\right), 7.63 \\
\left(\mathrm{~d}, J=9.6 \mathrm{~Hz}, 2 \mathrm{H}, 2-\mathrm{H}_{\mathrm{Ph}}\right), 7.80-8.10(\mathrm{~m}, 7 \mathrm{H}, \mathrm{Naph})\end{array}$ & $\begin{array}{l}44,77,101,110,127 \\
137,153,167,195,237 \\
313,314,342,357\end{array}$ \\
\hline 5 eg & $\begin{array}{l}3134,3006,2871 \\
1616,1594,1523 \\
1453,1117,865,826 \\
747\end{array}$ & $\begin{array}{l}2.79\left(\mathrm{~s}, 6 \mathrm{H}, \mathrm{NMe}_{2}\right), 3.15(\mathrm{dd}, J=15.5,8.1 \mathrm{~Hz}, 1 \mathrm{H}, \\
\left.\mathrm{CH}_{2 \text { (Pyraz) }}\right), 3.71\left(\mathrm{dd}, J=15.5,11.2 \mathrm{~Hz}, 1 \mathrm{H}, \mathrm{CH}_{\text {Pyraz }}\right), \\
5.09\left(\mathrm{dd}, J=11.2,8.1 \mathrm{~Hz}, 1 \mathrm{H}, \mathrm{CH}_{2(\text { (Praz) }}\right), 6.30- \\
8.20(\mathrm{~m}, 16 \mathrm{H}, \mathrm{Ar})\end{array}$ & $\begin{array}{l}48,64,77,91,120,121 \\
134,147,153,171,244 \\
271,389,391,392,393\end{array}$ \\
\hline $5 \mathrm{eh}$ & $\begin{array}{l}3120,2818,1660 \\
1635,1600,1589 \\
1348,1150,845,650\end{array}$ & $\begin{array}{l}2.81\left(\mathrm{~s}, 6 \mathrm{H}, \mathrm{NMe}_{2}\right), 3.26(\mathrm{dd}, J=15.0,8.2 \mathrm{~Hz}, 1 \mathrm{H}, \\
\left.\mathrm{CH}_{2 \text { (Pyraz) }}\right), 3.82\left(\mathrm{dd}, J=15.0,12.2 \mathrm{~Hz}, 1 \mathrm{H}, \mathrm{CH}_{\text {Pyraz }}\right) \\
5.36\left(\mathrm{bs}, 2 \mathrm{H}, \mathrm{NH}_{2}\right), 5.68(\mathrm{dd}, J=12.2,8.2 \mathrm{~Hz}, 1 \mathrm{H}, \\
\left.\mathrm{CH}_{2 \text { (Pyraz) }}\right), 7.21\left(\mathrm{~d}, J=10.3 \mathrm{~Hz}, 2 \mathrm{H}, 3-\mathrm{H}_{\mathrm{Ph}}\right), 7.58(\mathrm{~d}, \\
\left.J=10.3 \mathrm{~Hz}, 2 \mathrm{H}, 2-\mathrm{H}_{\mathrm{Ph}}\right), 7.70-8.10(\mathrm{~m}, 7 \mathrm{H}, \mathrm{Naph})\end{array}$ & $\begin{array}{l}42,77,120,127,147,153 \\
154,169,191,195,314,358\end{array}$ \\
\hline
\end{tabular}

\section{References}

1. Taylor, E. C.; Patel, H.; Kumar, H. Tetrahedron 1992, 48, 8089.

2. $\quad$ Roelfvan, S. G.; Arnold, C.; Wellnga, K. J. Agric. Food Chem. 1979, 84, 406.

3. Keats, G. H. Brit. Pat. 1,209,631, 1970.

4. Kedar, R. M.; Vidhale, N. N.; Chincholkar, M. M. Orient. J. Chem. 1997, 13, 143.

5. Singh, A.; Rathod, S.; Berad, B. N.; Patil, S. D.; Dosh, A. G. Orient. J. Chem. 2000, 16, 315.

6. Katri, H. Z.; Vunii, S. A. J. Ind. Chem. Soc. 1981, 58, 168.

7. $\quad$ Das, N. B.; Mittra, A. S. Ind. J. Chem. 1978, 16B, 638.

8. Azarifar, D.; Shaebanzadeh, M. Molecules 2002, 7, 885.

9. Holla, B. Shivarama; Akberali, P. M.; Shivanada, M. K. Farmaco 2000, 55, 256.

10. Palaska, E.; Aytemir, M.; Tayfun, I.; Erol, K. Dilek, E. Eur. J. Med. Chem. Chim. Ther. 2001, 36, 539.

11. Garge, H. G.; Chandraprakash, J. Pharm. Sc. 1971, 14, 649.

12. Regaila, H. A.; El-Bayonk, A. K.; Hammad, M. Egypt. J. Chem. 1979, 20, 197.

13. Krishna, R.; Pande, B. R.; Bharthwal, S. P.; Parmar, S. S. Eur. J. Med. Chem. 1980, 15, 567.

14. Husain, M. I.; Shukla, S. Ind. J. Chem. 1986, 25B, 983.

15. Tomilovi, Yu. V.; Okonnishnikova, G. P.; Shulishov, E. V.; Nefedov, O. M. Russ. Chem. Bt. 1995, 44, 2114. 
16. Klimova, E. I.; Marcos, M.; Klimova, T. B.; Cecilio, A. T.; Ruben, A. T.; Lena, R. R. J. Organomet. Chem. 1999, 585, 106.

17. Bhaskarreddy, D.; Padmaja, A.; Ramanareddy, P. V.; Seenaiah, B. Sulfur Lett. 1993, 16, 227.

18. Padmavathi, V.; Sumathi, R. P.; Chandrasekhar, B. N.; Bhaskarreddy, D. J. Chem. Research 1999, 610.

19. Bhaskarreddy, D.; Chandrasekhar, B. N.; Padmavathi, V.; Sumathi, R. P. Synthesis, 1998, 491.

20. Knorr, L. Ber. Dt. Chem. Ges. 1893, 26, 100.

21. Thakare, V. G.; Wadodkar, K. N. Ind. J. Chem. Sect. B 1986, 25, 610.

22. Ankhiwala, M. D.; Hathi, M. V. J. Ind. Chem. Soc. 1994, 71, 587.

23. Vounauwers, K.; Muller, A. Ber. Dt. Chem. Ges. 1908, 41, 4230.

24. Baker, A.; Butt, V. S. J. Chem. Soc. 1949, 2142.

25. Kadu, V. B.; Dashi, A. G. Orient. J. Chem. 1997, 13, 285.

26. Abdelhamid, A. O.; Zohdi, H. F.; Sallam, M. M. M.; Ahmed, N. A. Molecules, 2000, 5, 967.

27. Rurack, K.; Bricks, J. L.; Schultz, B.; Maus, M.; Reck, G.; Resch-Genger, U. J. Phys. Chem. A. 2000, 104, 6171.

28. Guerra, F. M.; Mish, M. R.; Carreira, E. M. Org. Lett. 2000, 2, 4265.

29. Molteni, G.; Garanti, L. Heterocycles 2001, 55, 1573.

30. Yadav, J. S.; Reddy, B. V. S.; Greetha, V. Syn. Lett. 2002, 3, 513.

31. Nakamichi, N.; Kawashita, Y.; Hayashi, M. Org. Lett. 2002, 4, 3955.

32. Wang, S.; Shi, B.; Li, Y.; Wang, Q.; Huang, R. Synth. Commun. 2003, 33, 1449.

33. Chen, Y.; Lam, Y.; Lai, Y. H. Org. Lett. 2003, 5, 1067.

Sample Availability: Available from the authors.

(C) 2003 by MDPI (http://www.mdpi.org). Reproduction is permitted for noncommercial purposes. 